\title{
NEW ASPECTS IN THE THEORY OF AQUEOUS ELECTROLYTE SOLUTIONS: THE EFFECTS OF CATION HYDROLYSIS AND POLYNUCLEAR ION FORMATION
}

\author{
M.F.HolovkO \\ Institute for Condensed Matter Physics, \\ National Academy of Sciences of Ukraine, \\ 1 Svientsitskii St., UA-290011 Lviv-11, Ukraine
}

Received December 30, 1997

\begin{abstract}
The polynuclear ion formation in aqueous solutions of metal salts is mimiced by the intercationic association interaction. The statistical mechanics of such ionic model is solved using multidensity associative formalism. The theory predicts a significant influence of the polynuclear ion formation on the thermodynamics and structure properties of electrolyte solutions in a wide range of concentrations including very dilute concentration regions.
\end{abstract}

\section{Introduction}

Aqueous electrolyte solutions are ion-molecular systems rather complicated for theoretical treatment. Water actually plays a double role here. It behaves as a solvent with a high static dielectric constant which favors the dissociation of ionic species. On the other hand, water is a $\sigma$-donor molecule which reacts as a nucleophilic ligand with ions. Such ion-water chemical reactions are known as hydrolysis and convert ions into new ionic species. Among those new species polynuclear ions are formed due to the partial hydrolysis of the aqueous solution of polyvalent metal cations, such as some elements belonging to the $\mathrm{p}\left(\mathrm{B}^{3+}, \mathrm{Al}^{3+}\right), \mathrm{d}\left(\mathrm{Fe}^{3+}, \mathrm{Zr}^{4+}, \mathrm{Cr}^{6+}, \ldots\right)$ or $\mathrm{f}$ (such lanthanides and actinides as $\left.\mathrm{Tr}^{4+}, \mathrm{U}^{4+}, \mathrm{Pu}^{4+}, \ldots\right)$ groups [1]. The nature of these species is of the fundamental interest in inorganic solution chemistry and of particular importance in many areas ranging from nuclear technology (gel fuel, radioactive contamination, aqueous corrosion) to environmental chemistry (soil science, mineral dissolution, etc.) [2]. Polynuclear ions are frequently the precursors of the formation of larger colloidal oxide particles, also known as sols which are of considerable technological importance [3]. The investigation of polynuclear ions is also important in connection with the Chornobyl accident, which enables the nuclear fuel to penetrate outside the reactor. As a result, the formation of aqueous solutions with such actinides as $\mathrm{U}$ and $\mathrm{Pu}$ becomes possible [4].

The hydrolysis of metal cations was first studied by N.Bjerrum at the beginning of the 20th century [5]. However, despite their considerable importance, studies of polynuclear ions in aqueous solutions with the use of modern physico-chemical techniques have been scarce. A certain limited amount of information about the size, structure and diffusion behaviour of polynuclear ions was obtained during the last decade from a combination of light and neutron scattering techniques [6]. Up till now the formation of polynuclear hydrolysis products in theoretical studies has almost been

(c) M.F.Holovko, 1997

ISSN 0452-9910. Condensed Matter Physics 1997 No 12 (13-25) 
ignored. In the traditional ion approach general attention is focused on the account of the effects of ionic screening, the size of ions and ionic pairing [7-8]. In a more consistent ion-molecular approach the attention is focused on the explicit consideration of the influence of the solvent structure on the properties of electrolyte solutions, the investigation of the role of hydrogen bonds and consistent description of ionic solvation effects [8-10]. However, the effects of hydrolysis has never been discussed in the both theoretical approaches.

The modelling of the influence of hydrolysis and polynuclear ion formation effects on the properties of aqueous electrolyte solutions within the framework of the ionic approach will be the subject of the present article. In our study we will consider the simplest primitive electrolyte model (the model of charged hard spheres) with an additional associative interaction between cations. This associative interaction is specified in such a way that cations will form linear chains or more complex polynuclear ions upon association. The description of this model will be carried out within the framework of the multidensity formalism developed recently in the theory of associating fluids [11-14]. We will show that the influence of the polynuclear ion formation on the thermodynamics and structure properties of electrolyte solutions is important including a very dilute ionic concentration region.

The article is organized as follows. The mechanisms of cation hydrolysis and polynuclear ion formation in aqueous solution of metal salts are reviewed in the second section. In the third section an associative ionic model for the electrolyte with polynuclear ions is introduced and theoretical methods of the description of this model are presented. In the fourth section the discussion of the results is given. In the last section our conclusions are formulated.

\section{Cation hydrolysis and polynuclear ion formation}

Hydrolysis or, more general, solvolysis is a substitution reaction of the solute with the solvent. The cation hydrolysis involves a stepwise process which begins within the creation of solvated aquo-ions according to the following scheme:

$$
\mathrm{M}^{Z+}+n \mathrm{H}_{2} \mathrm{O} \longleftrightarrow\left[\mathrm{M}\left(\mathrm{H}_{2} \mathrm{O}\right)_{n}\right]^{Z+}
$$

where $Z^{+}$is the valency of cation $M^{Z+}$ and $\mathrm{n}$ is the number of water molecules bounded to the cation (hydration number).

The solvation of the metal ions of $p, d$ or $f$ groups leads to the formation of a partially covalent bond. A partial charge transfer occurs from the bonding orbital of the water molecule to the empty $p, d$ or $f$ orbitals of the cation. The positive partial charge on the hydrogen atoms then increases and water becomes more acidic. The first step of hydrolysis leads to the creation of the mononuclear hydroxo ions

$$
\left[M\left(\mathrm{H}_{2} \mathrm{O}\right)_{n}\right]^{Z+}+\mathrm{H}_{2} \mathrm{O} \longleftrightarrow\left[M\left(\mathrm{H}_{2} \mathrm{O}\right)_{n-1} \mathrm{OH}\right]^{(Z-1)+}+\mathrm{H}_{3} \mathrm{O}^{+}
$$

Usually the process does not stop at this step and, as a result of the condensation reaction, polynuclear ions appear [15]:

$$
p\left[M\left(H_{2} O\right)_{n}\right]^{Z+}+q H_{2} O \longleftrightarrow\left[M_{p}(O H)_{q} H_{2} O_{p n-q}\right]^{(p Z-q)+}+q H_{3} O^{+}
$$




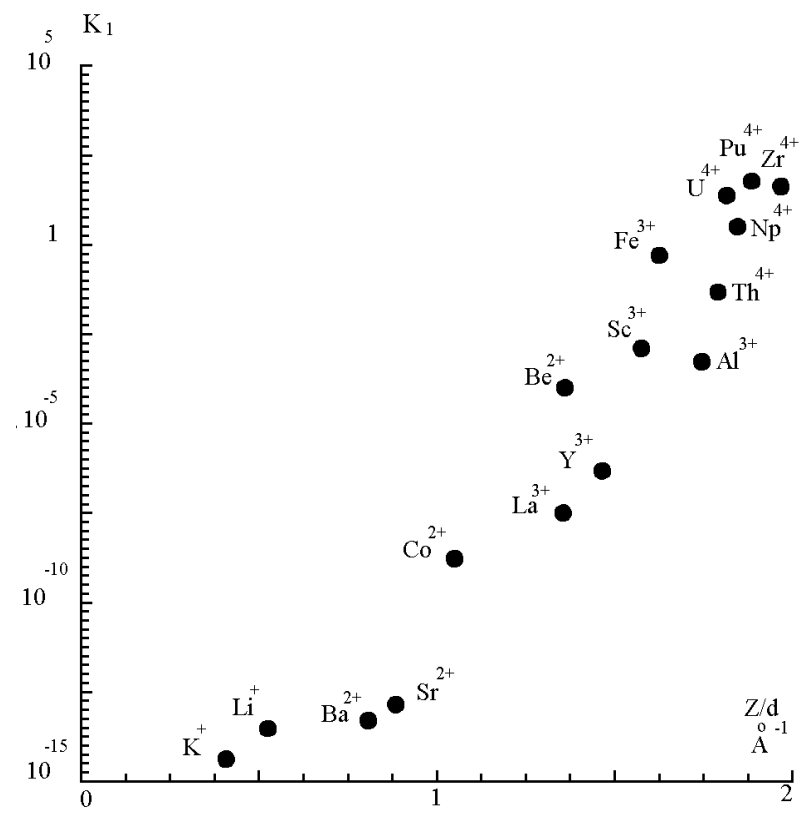

Figure 1. Dependence of the first hydrolysis constant $K_{1}$ on the ratio of the charge to the $\mathrm{M}-\mathrm{O}$ distance for different cations.

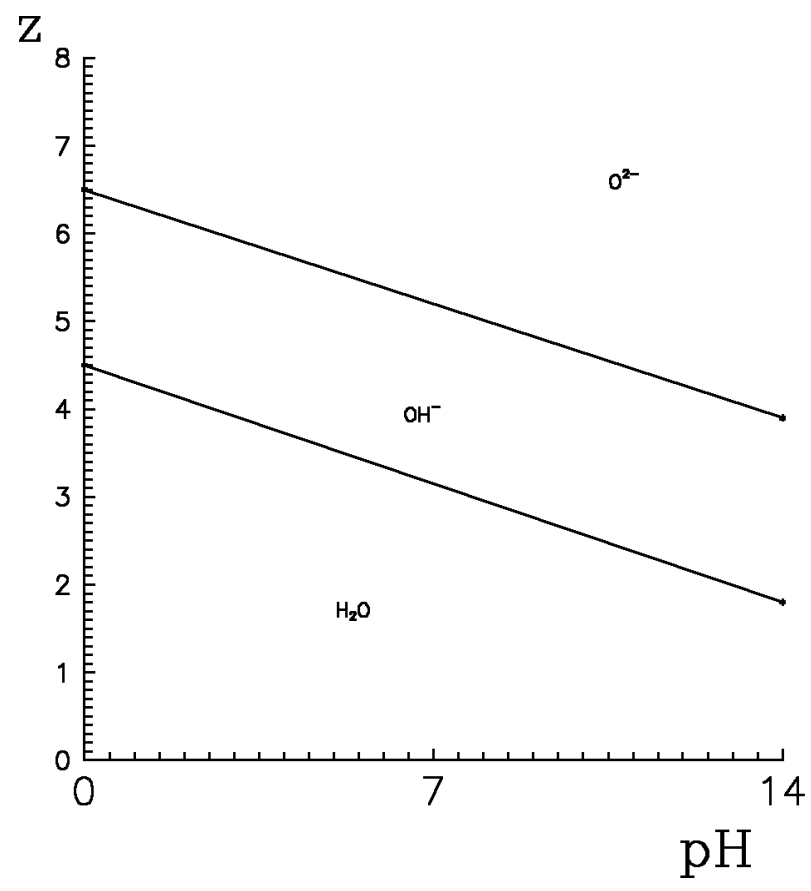

Figure 2. The charge-pH diagram. 
Such a sequence of hydrolytic and condensation reactions leads, under the conditions of the metal hydroxide over-saturation, to the formation of colloidal hydroxy polymers which can eventually form hydroxide or hydrous oxide precipitates. This tendency for the cation hydrolysis involving the coordination of $\mathrm{OH}$ ligands, which act as electron pair donors, increases with the acidity or charge density of the metal ion. Consequently, ions of a small ionic radius and high charge are extensively hydrolysed in the solution. The tendency to hydrolysis can be generalized for a wide range of cations, as demonstrated in figure 1. [6] In this figure the dependence of the first hydrolysis constant $K_{1}$ (for reaction 2.2) on the ratio of the charge to the $\mathrm{M}-\mathrm{O}$ distance $(\mathrm{Z} / \mathrm{d})$ is shown. The tendency to the cation hydrolysis is also paralleled by the ease of the polynuclear ion formation. Thus, most trivalent and quadrivalent ions form polynuclear species, whereas this process only occurs with $\mathrm{Be}^{2+}$, the smallest of the divalent ions.

As a result of the hydrolysis, with the increase of the acidity or charge density of the cation, the oxo-ions which have only M-O bonds can appear. In general, the cation hydrolysis converts solvated ion $\left[\mathrm{M}\left(\mathrm{H}_{2} \mathrm{O}\right)_{n}\right]^{Z+}$ into precursor $\left[M O_{n} H_{2 n-h}\right]^{(Z-h)+}$, where $\mathrm{h}$ is defined as a molar ratio of the hydrolysis [3]. The precursor can be positive for $Z>h$, neutral for $Z=h$ and even negative for $Z<h$. When $h=0$ the ion has the usual aquo-ion $\left[M\left(\mathrm{H}_{2} \mathrm{O}\right)_{n}\right]^{Z+}$ form, while for $\mathrm{h}=2 \mathrm{n}$ it is oxo-ion $\left[\mathrm{MO}_{n}\right]^{(2 n-Z)-}$. If $0<h<$ $2 n$, the precursor can be either oxo-hydroxo ion $\left[\mathrm{MO}_{h-n}(\mathrm{OH})_{2 n-h}\right]^{(h-Z)-}$ $(h>n)$, hydroxo-aquo ion $\left[\mathrm{M}(\mathrm{OH})_{h}\left(\mathrm{OH}_{2}\right)_{n-h}\right]^{(Z-h)+}(h<n)$ or hydroxo ion $\left[M(O H)_{n}\right]^{(n-Z)-}(\mathrm{h}=\mathrm{n})$. The value of $\mathrm{h}$ strongly depends on the $\mathrm{pH}$ and charge of the cation. This fact is demonstrated in the charge - $\mathrm{pH}$ diagram shown in figure 2. This diagram shows the nature of the precursor as a function of the formal charge $\mathrm{Z}$ of the cation $M^{Z+}$ and $\mathrm{pH}$ of the aqueous solution. Three domains can be defined, namely: aquo $\left[\mathrm{M}\left(\mathrm{OH}_{2}\right)_{n}\right]^{Z+}$, hydroxo $\left[M O_{n} H_{2 n-h}\right]^{(Z-h)+}$, and oxo $\left[M O_{n}\right]^{(2 n-Z)+}$. The diagram shows that low-valent cations $(Z<+4)$ give rise to aquo-hydroxo and/or hydroxo ionic complexes over the whole range of $\mathrm{pH}$, while high-valent cations $(Z>+5)$ form oxo-hydroxo and/ or oxo ionic complexes over the same range of $\mathrm{pH}$. Tetravalent cations $(\mathrm{Z}=+4)$ are on the border line, and, therefore, lead to a large number of possible precursors.

The precursor $\left[M O_{n} H_{2 n-h}\right]^{(Z-h)+}$ is a structural unit for polynuclear ions and in the present theoretical description we consider them as ions with valency $z=Z-h$. By varying the chemical conditions under which cations are polymerized, different structures can be formed which range from randomly branched polymers to colloidal particles. However, for the simplification in this article our consideration will be limited to the simplest case of polynuclear cation chain formation.

\section{The model and theory}

In this article the description of aqueous electrolyte solutions is based on the ionic approach in which only the ionic subsystem is considered explicitly. In our approach the ions are represented by ionic complexes $\left[\mathrm{MO}_{n} \mathrm{H}_{2 n-h}\right]^{(Z-h)+}$ formed as a result of hydrolysis. The effect of solvent molecules is accounted for semiphenomenologically by introducing the macroscopic dielectric constant $\varepsilon$ into the Coulomb interaction law and by appropriately choosing the short-range part of the ionic interaction. For the description of the hydrolysis effects we introduce an additional associative interaction between 
cations which leads to the formation of polynuclear ions. As a result, the pair interionic interaction includes three terms

$$
U_{a b}^{\mathrm{sh}}(r)=U_{a b}^{\mathrm{sh}}(r)+U_{a b}^{\mathrm{el}}(r)+U_{a b}^{\mathrm{as}}(r),
$$

where $U_{a b}^{\mathrm{sh}}(r)$ is a short-range part of the interionic interaction, $U_{a b}^{\mathrm{el}}=\frac{e^{2} z_{a} z_{b}}{\varepsilon r}$ is the Coulomb term and $U_{a b}^{\text {as }}(r)$ is the association term, $e z_{a}$ is the ionic charge.

For simplification we are using a restricted primitive electrolyte model in which the system is represented by the two-component charged hard sphere mixture with valencies $z_{+}=-z_{-}=1$ and with diameters $d_{+}=d_{-}=d$. Due to electroneutrality the concentrations $c_{+}=c_{-}=\frac{1}{2} c$. We assume that associative interaction $U_{a b}^{\text {as }}(r)$ exists only between cations and that each positively charged hard sphere has two attractive sticky points, A and $\mathrm{B}$, randomly placed on the surface. In addition we assume that the probability of binding the two sites forming a certain angle $\alpha_{A B}$ between the vectors connecting the corresponding site and the center of the hard sphere is independent of $\alpha_{A B}$

$$
U_{++}^{\text {as }}(r)=U_{++}^{A B}(r)+U_{++}^{B A}(r), U_{+-}^{\text {as }}(r)=U_{--}^{\text {as }}(r)=0
$$

where $U_{++}^{A B}$ are the site-site potentials representing the sticky interaction.

Due to the random location of the attractive sites, the model at hand represents a model for associating particles with positively charged ions that polymerize into the freely-joined tangent charged hard-sphere linear chain ions. Description of the model in question can be carried out within the framework of the modern theory of associating fluids [11-14] which incorporates two types of the calculation methods: the thermodynamic perturbation theory for the free energy and the integral equation for correlation functions.

\subsection{Thermodynamic perturbation theory.}

In this approach the restrictive primitive electrolyte model is considered to be the reference system and the association contribution is treated using the thermodynamic perturbation theory. As a result, the free energy can be represented as a sum of four terms:

$$
A=A^{\mathrm{id}}+A^{\mathrm{hs}}+A^{\mathrm{el}}+A^{\text {bound }},
$$

where $A^{\text {id }}$ is an ideal term, $A^{\text {hs }}$ is a hard sphere contribution, $A^{\text {el }}$ is an ionic term and $A^{\text {bound }}$ is an association contribution.

We also have the same form for the pressure and chemical potentials of the system

$$
\begin{gathered}
\frac{p}{c k T}=c \frac{\partial}{\partial c}\left(\frac{A}{N k T}\right)=\frac{\left(p^{\mathrm{hs}}+p^{\mathrm{el}}+p^{\mathrm{bound}}\right)}{c k T} \\
c_{a} \mu_{a}=c_{a} \frac{\partial}{\partial c_{a}}\left(\frac{A}{V}\right)=c_{a}\left(\mu_{a}^{\mathrm{id}}+\mu_{a}^{\mathrm{hs}}+\mu_{a}^{\mathrm{el}}+\mu_{a}^{\mathrm{bound}}\right) .
\end{gathered}
$$

The hard sphere and electrostatic contributions are given by the Carnahan-Starling equation and by the mean spherical approximation correspondingly [8]. 


$$
\begin{gathered}
\frac{A^{\mathrm{hs}}}{N k T}=\frac{4 \eta-3 \eta^{2}}{(1-\eta)^{2}}, \quad \frac{p^{\mathrm{hs}}}{c k T}=\frac{1+\eta+\eta^{2}-\eta^{3}}{(1-\eta)^{3}}, \\
\frac{\mu^{\mathrm{hs}}}{k T}=\frac{8 \eta-9 \eta^{2}+3 \eta^{3}}{(1-\eta)^{3}}, \\
\frac{A^{\mathrm{el}}}{N k T}=-\frac{\beta e^{2} z^{2}}{\varepsilon} \frac{\Gamma}{1+\Gamma d}+\frac{\Gamma^{3}}{3 \pi c}, \\
\frac{p^{\mathrm{el}}}{c k T}=-\frac{\Gamma^{3}}{3 \pi c}, \quad \frac{\mu^{\mathrm{el}}}{k T}=-\frac{\beta e^{2} z^{2}}{\varepsilon} \frac{\Gamma}{1+\Gamma d},
\end{gathered}
$$

where $\eta=\frac{1}{6} \pi c d^{3}$ is the packing fraction, $2 \Gamma d=\sqrt{1+2 \kappa d}-1$, $\kappa=\left(4 \pi c e^{2} z^{2} /(\varepsilon k T)\right)^{1 / 2}$ is the Debye-Huckel inverse screening length.

The associative contribution is made by the thermodynamic perturbation theory [11-12]

$$
\begin{array}{r}
\frac{A^{\text {bound }}}{N k T}=2\left(\frac{m-1}{2 m}-\ln m\right), \quad \frac{p^{\text {bound }}}{c k T}=-\frac{2 m-1}{m^{2}} c \frac{\partial m}{\partial c}, \\
\mu_{+}^{\text {bound }}=2\left(\frac{m-1}{2 m}-\ln m-\frac{1}{2} \frac{2 m-1}{m^{2}} c \frac{\partial m}{\partial c}\right) .
\end{array}
$$

where $\mathrm{m}$ is the mean chain length defined from the following equation:

$$
m(m-1)=2 \pi c K_{++} g_{o o}^{++}(d+),
$$

$K_{++}$is the stickiness parameter defined in such a way that the site-site potential is chosen to give the corresponding average Mayer function:

$$
\begin{gathered}
f_{++}^{A B}(r)=e^{-\beta U_{++}^{A B}(r)}-1=K_{++} \delta(r-d), \\
g_{++}^{o o}(d+)=g_{++}^{\mathrm{ref}}(d+)=\frac{1-\frac{1}{2} \eta}{(1-\eta)^{3}} \exp \left[-\beta \frac{e^{2} z^{2}}{\varepsilon d}\left(\frac{2 \Gamma}{\kappa}\right)^{2}\right]
\end{gathered}
$$

is the contact value of the cation-cation distribution function for the restrictive primitive model.

\subsection{The integral equation theory}

The integral equation theory for associative fluids is based on multidensity version of the Ornstein-Zernike (OZ) equation [13-14]. In the case of associating cations with independent attraction sites the correlation functions can be orientationally-averaged independent over the orientation of each attractive site. The orientationally-averaged version of the multidensity $\mathrm{OZ}$ equation can be written in terms of the orientationally- averaged partial correlation functions $h_{a b}^{\alpha \beta}(r)$ and $c_{a b}^{\alpha \beta}(r)$

$$
\mathbf{h}_{a b}(r)=\mathbf{c}_{a b}(r)+\sum \int \mathbf{c}_{a c}\left(r^{\prime}\right) \mathbf{c}_{c} \mathbf{h}_{c b}\left(\left|\mathbf{r}-\mathbf{r}^{\prime}\right|\right) \mathbf{r}^{\prime},
$$


where $\mathbf{h}_{a b}(r)$ are the matrices defined by

$$
\begin{aligned}
\mathbf{h}_{++}(r)= & \left(\begin{array}{ccc}
h_{++}^{o o} & h_{++}^{o A} & h_{++}^{o B} \\
h_{+o}^{A o} & h_{+A}^{A A} & h_{+B}^{A B} \\
h_{++}^{B+} & h_{++}^{B A} & h_{++}^{B+B}
\end{array}\right), \quad \mathbf{h}_{-+}(r)=\left(\begin{array}{c}
h_{+-}^{o} \\
h_{+-}^{A} \\
h_{+-}^{B}
\end{array}\right) \\
& \mathbf{h}_{+-}(r)=\left(h_{-+}^{o}, h_{-+}^{A}, h_{-+}^{B}\right), \mathbf{h}_{--}(r)=h_{--}(r)
\end{aligned}
$$

and similarly for $\mathbf{c}_{a b}(r)$. The matrices $\mathbf{c}_{a}$ in the ideal chain approximation [14] are given by

$$
\mathbf{c}_{+}=\frac{1}{2} c\left(\begin{array}{ccc}
1 & 1 / m & 1 / m \\
1 / m & 0 & 1 / m^{2} \\
1 / m & 1 / m^{2} & 0
\end{array}\right), \quad \mathbf{c}_{-}=c_{-}=\frac{1}{2} c,
$$

where $m$ is the mean chain length defined by equation (3.9) with the contact value of cation-cation distribution function $g_{++}^{o o}(r)=1+h_{++}^{o o}(r)$ obtained self-consistently from the $\mathrm{OZ}$ equation. As a closure relation for the $\mathrm{OZ}$ equation (3.12) we are using the polymer hypernetted chain ( $\mathrm{P} \mathrm{H} \mathrm{N} \mathrm{C}$ ) approximation [16], relating the direct $c_{a b}^{\alpha \beta}(r)$ and total $h_{a b}^{\alpha \beta}(r)$ partial correlation functions as

$$
\begin{aligned}
& g_{++}^{o o}(r)=h_{++}^{o o}(r)+1=\exp \left(-\beta\left[U_{++}^{\mathrm{sh}}(r)+U_{++}^{\mathrm{el}}(r)\right]+\tau_{++}^{o o}(r)\right), \\
& g_{+-}^{o}(r)=h_{+-}^{o}(r)+1=\exp \left(-\beta\left[U_{+-}^{\mathrm{sh}}(r)+U_{+-}^{\mathrm{el}}(r)\right]+\tau_{+-}^{o}(r)\right), \\
& g_{--}(r)=h_{--}(r)+1=\exp \left(-\beta\left[U_{--}^{\mathrm{sh}}(r)+U_{--}^{\mathrm{el}}(r)\right]+\tau_{--}(r)\right), \\
& g_{++}^{o A}(r)=h_{++}^{o A}(r)=g_{++}^{o o}(r) \tau_{++}^{o A}(r), g_{+-}^{A}(r)=h_{+-}^{A}(r)=g_{+-}^{o}(r) \tau_{+-}^{A}(r), \\
& g_{++}^{A B}(r)=h_{++}^{A B}(r)= \\
& \quad g_{++}^{o o}(r)\left[\tau_{++}^{A B}(r)+\tau_{++}^{A o}(r) \tau_{++}^{o B}(r)+f_{++}^{A B}(r)+f_{++}^{B A}(r)\right], \\
& g_{++}^{A A}(r)=h_{++}^{A A}(r)=g_{++}^{o o}(r)\left[\tau_{++}^{A A}(r)+\tau_{++}^{A o}(r) \tau_{++}^{o A}(r)\right] .
\end{aligned}
$$

where $\beta=1 / k T, \tau_{a b}^{\alpha \beta}(r)=h_{a b}^{\alpha \beta}(r)-c_{a b}^{\alpha \beta}(r)$. The indices $\alpha$ and $\beta$ denote the bonded states of the corresponding particle. The case of $\alpha=0$ corresponds to an unbounded particle, $\alpha=A$ or $\alpha=B$ - to the particle with the bonded site $\mathrm{A}$ or $\mathrm{B}$.

In the present theory the equilibrium properties of the system are described via the average pair distribution functions $g_{a b}(r)$ which are related to the partial distribution functions $g_{a b}^{\alpha \beta}(r)$ by

$$
\begin{array}{r}
g_{++}(r)=g_{++}^{o o}(r)+\frac{2}{m}\left[g_{++}^{o A}(r)+g_{++}^{A o}(r)\right]+\frac{2}{m^{2}}\left[g_{++}^{A B}(r)+g_{++}^{A A}(r)\right], \\
g_{+-}(r)=g_{+-}^{o}(r)+\frac{2}{m} g_{+-}^{A}(r),
\end{array}
$$

Thermodynamical properties of the system can be obtained by using the same expressions as those in section 3.1 with $\mathrm{m}$ calculated self-consistently together with the solution of the $\mathrm{OZ}$ equation. 


\section{Results and discussion}

It is known $[7,8]$ that the Coulomb interionic interaction defines the behaviour of usual electrolyte solutions for the dilute range of ionic concentrations. The influence of the Coulomb interaction is characterized by two parameters: the Debye length $r_{\mathrm{D}}=\kappa^{-1}$ and the Bjerrum length $r_{\mathrm{B}}=\beta e^{2} z^{2} \frac{1}{\varepsilon}$ which describe the ionic screening and ionic pairing effects correspondingly. The association intercationic interaction includes a new parameter $\mathrm{m}$ which characterizes the mean length value of the polynuclear ionic chain formation. For the small ionic concentration according to (3.9) $m \sim \sqrt{c}$ and has concentration dependence similar to the inverse Debye length $\kappa \sim \sqrt{c}$. Depending on the value of $m$ the effect of the polynuclear ions formation can be significant and can lead to a strong deviation from the Debye-Huckel limiting behaviour for the osmotic and activity coefficients

$$
\Phi=\frac{p}{c k T}, \quad \ln \gamma_{+-}=\frac{1}{2} \beta\left[\mu_{+}-\mu_{+}^{\mathrm{id}}+\mu_{-}-\mu_{-}^{\mathrm{id}}\right] .
$$

Using the theory presented in the previous section, we have evaluated the influence of cation hydrolysis and polynuclear ion formation on the thermodynamic and structure properties of aqueous electrolyte solutions. Both the thermodynamic perturbation theory and the integral equation theory yield qualitatively similar thermodynamic properties. The integral equation method needs more numerical efforts in comparison with the thermodynamic perturbation theory. But it gives, in addition to the thermodynamic properties, the pair distribution functions which characterize the structure of the ionic subsystem. The numerical solution of the OZ equation in PHNC approximation is obtained using the direct iteration method in [16]. Below we consider only some results obtained in [16] to illustrate the influence of hydrolysis on the properties of aqueous electrolyte solutions.

We consider here two examples which correspond to the polynuclear ion formation for cations $\mathrm{Al}^{3+}$ and $\mathrm{Zr}^{4+}$. The properties of other solutions of metal salts will be similar. For example, since the first hydrolysis constant $\mathrm{U}^{4+}$ and $\mathrm{Pu}^{4+}$ has nearly the same value as for $\mathrm{Zr}^{4+}$ (see figure 1) we can expect that aqueous solutions with these actinides will have the similar properties as the solution with $\mathrm{Zr}^{4+}$. As it was shown by Ramsay [6] from the light and neutron scattering measurements on relatively concentrated solutions (more than 0.1 mole/1) ions $\mathrm{Al}^{3+}$ create chains with the length nearly 30 ions and ions $\mathrm{Zr}^{4+}$ create chains containing approximately 200 ions. From this data we fit the stickiness parameter $\mathrm{K}_{++}$of the intercationic association and assume that at 1 mole $/ 1 m_{\mathrm{Al}^{3+}}=30$ and $m_{\mathrm{Zr}^{4+}}=200$. The dependence of the mean chain length $\mathrm{m}$ on the ionic concentration is illustrated in figure 3 One can see that with the increase of the ionic concentration the value of $\mathrm{m}$ increases. Our calculations show that at the concentration 0.001 mole $/ 1 m_{\mathrm{Al}^{3+}} \simeq 3$ and at the concentration $10^{-6}$ mole/l $m_{\mathrm{Zr}^{4+}} \simeq 2$. For a more dilute solution, according to (3.9), $m \rightarrow 1$ and only mononuclear ions exist.

The influence of the polynuclear ion formation on the activity and osmotic coefficients is illustrated in figure 4 . One can see that with the increase of the interactionic association the deviations from the behaviour of the restrictive primitive model strongly increase, which is explained by increasing the mean chain length of polynuclear ions. With the increase of the ionic concentration the value of $\mathrm{m}$ also increases and a deviation from the ionic restrictive primitive model behaviour increases, however more slowly for 


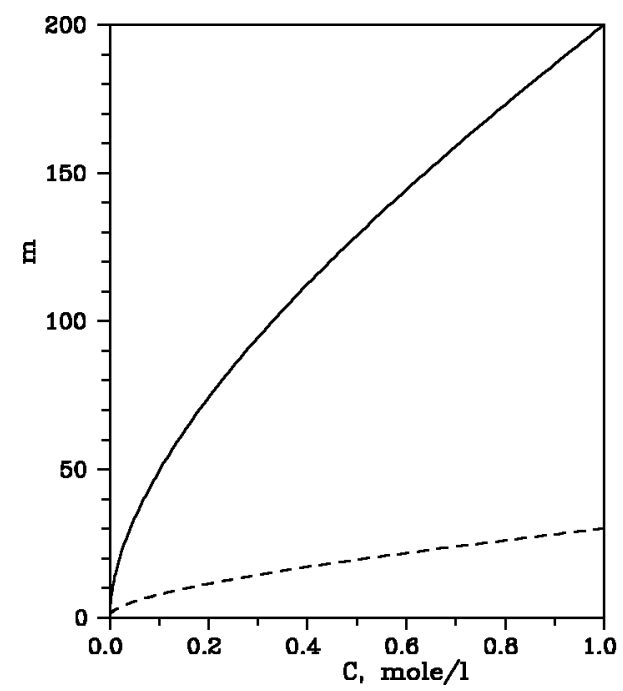

Figure 3. The mean chain length $\mathrm{m}$ of polynuclear ions as a function of ionic concentration $c$.

- . - . - solution with ions $\mathrm{Al}^{3+}$, - solution with ions $\mathrm{Zr}^{4+}$.

higher concentrations due to the influence of the hard sphere contribution which does not depend on the degree of association.

The influence of the polynuclear ion formation on the ionic structure is more pronounced. The pair distribution functions of the restrictive primitive electrolyte model for the concentration region smaller than 1 mole/l are monotonous enough and $g_{++}(r)=g_{--}(r)$ [8]. Due to the formation of polynuclear ions the intraionic distribution function

$$
g_{++}^{\text {intra }}(r)=\frac{1}{m^{2}}\left[g_{++}^{A B}(r)-g_{++}^{A A}(r)\right]=\frac{1}{m^{2}} g_{++}^{o o}(r)\left(f_{++}^{A B}(r)+f_{++}^{B A}(r)\right)
$$

appears in the cation-cation distribution function $g_{++}(r)$ and according to (3.9) the cation-cation coordination number

$$
n_{++}=2 \pi c \int_{0}^{\infty} g_{++}^{\text {intra }} r^{2} \underline{r}=2 \frac{m-1}{m}
$$

is fixed. As a result, the first maximum of the pair distribution function $g_{++}(r)$ increases with the increase of chain length $\mathrm{m}$. According to condition (4.3) the first maximum of $g_{++}(r)$ for large enough $m$ will also increase with the decrease of the ionic concentration.

Such a change of $g_{++}(r)$ produces corresponding changes for all pair distribution functions which are connected by the local electroneutrality condition $[8]$

$$
2 \pi c \int_{0}^{\infty}\left[g_{+-}(r)-g_{++}(r)\right] r^{2} \stackrel{\varphi}{\varphi}=2 \pi c \int_{0}^{\infty}\left[g_{+-}(r)-g_{--}(r)\right] r^{2} \stackrel{\varphi}{r}=1
$$



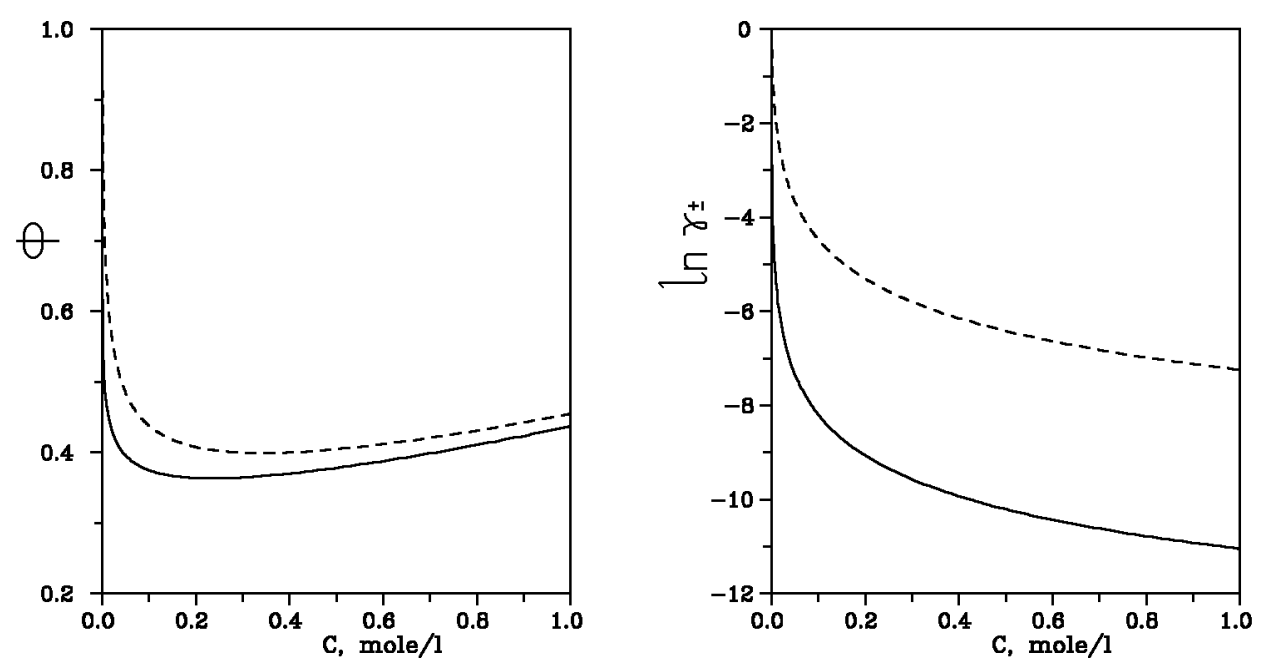

Figure 4. The osmotic coefficient $\Phi$ and the mean ionic activity coefficient $\gamma_{ \pm}$for aqueous solutions of metal salts. - . - . - solution with ions $\mathrm{Al}^{3+}$, - - solution with ions $\mathrm{Zr}^{4+}$.

As a result of the polynuclear ion formation the equality $g_{++}(r)=g_{--}(r)$ is broken and instead of it, according to (4.4), we have

$$
\int_{0}^{\infty}\left[g_{++}(r)-g_{--}(r)\right] r^{2} \underline{r}=0
$$

Since all the distribution functions are connected by the local electroneutrality conditions, a change in one of them causes changes in all the other distribution functions. Here we discuss such changes connected with the polynuclear ion formation. The influence of the polynuclear ion formation on the ionic structure is illustrated in figure 5 in which pair distribution functions for two values of the ionic concentration 0.01 mole/ 1 and 0.105 mole/l are shown. For comparison the pair distribution functions for the restrictive primitive model $(m=1)$ are also presented here. One can see that the appearance and the increase of the first maximum of $g_{++}(r)$ produces the increase of the first maximum of the cation-anion distribution function $g_{+-}(r)$ which leads to the specific "condensation" of anions into polynuclear ions. One can also observe changes in the behaviour of the anion-anion distribution function $g_{--}(r)$ which becomes more structural with the increase of $\mathrm{m}$. 

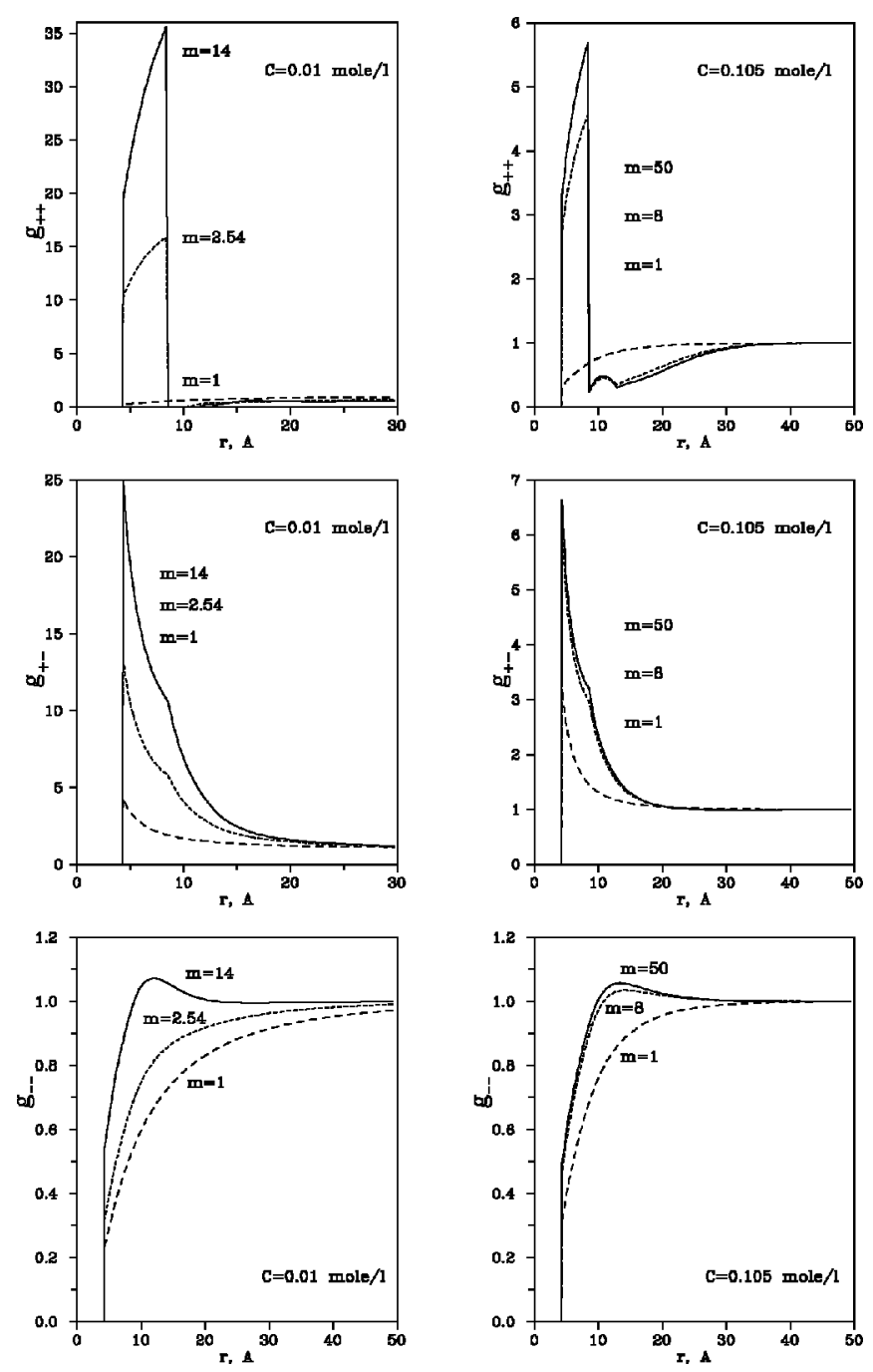

Figure 5. Ionic pair distribution function for aqueous electrolyte solutions.

$-{ }_{-}-{ }_{-}-$primitive electrolyte model $(m=1)$,

- . - . - solution with ions $\mathrm{Al}^{3+}$,

- solutions with ions $\mathrm{Zr}^{4+}$. 


\section{Conclusions}

In this paper the mechanisms of the cation hydrolysis and polynuclear ion formation in aqueous solutions of metal salts are reviewed. For the description of the influence of this phenomena on the properties of electrolyte solutions we expand usual ionic models by including the additional associative interaction between cations which leads to the formation of polynuclear ions. Our approach is based on the multidensity formalism developed in the modern theory of associating fluids.

The influence of polynuclear ion formation is characterized by the mean chain length $\mathrm{m}$ which has the similar dependence on the ionic concentration as the inverse Debye length $\kappa$. We have shown that the influence of the polynuclear ion formation on thermodynamics and structure properties of electrolyte solutions can be very important in a wide range of concentrations, including a rather dilute concentration region of $10^{-6} \mathrm{~mole} / \mathrm{l}$, when polynuclear ions begin to form.

As a result of the formation of polynuclear ions, the intracationic distribution function $g_{++}^{\text {intra }}(r)$ appears in the cation-cation distribution function $g_{++}(r)$. Since all the distribution functions are connected due to the local electroneutrality condition, such a change of $g_{++}(r)$ automatically leads to the corresponding changes of $g_{+-}(r)$ and $g_{--}(r)$.

\section{Acknowledgments}

This work was supported in part by the joint INTAS-Ukraine Call'95 grant (INTAS-UA95-133). I am very indebted to Yu. Kalyuzhnyi for his careful reading the manuscript and preparation of figures 4-5.

\section{References}

[1] Baes C.F., Messmer R.E. The hydrolysis of cations. New York, Wiley Interscience, 1976.

[2] Stumm W., Morgan J. Aquatic chemistry, New York, Wiley Interscience, 1981.

[3] Livage J., Henry M., Sanchez C. Sol-gel chemistry of transition metal oxides. Progr. Solid State Chem. 18, p. 259-341, 1988.

[4] Yukhnovsky I.R., Tokarchuk M.V., Ignatyuk V.V., Kobryn A.E., Omelyan I.P., Zhelem R.J., Dmytriv G.S., Ivankiv O.L. Studies on nonequilibrium physico-chemical process in the system "fuel containing masses - aqueous solutions of radioactive elements" // Cond. Matt. Phys., 1997, vol. 12, p. 63-96.

[5] Bjerrum N. // Z. Phys. Chem, 59, 336, 1907.

[6] Ramsay J.D.F. Neutron and light scattering studies of aqueous solutions of polynuclear ions in water and aqueous solutions. Ed. by Neilson G.W. and Enderby J.E. Bristol, Adam Hilger, 1986, p. 207-218.

[7] Falkengagen H., Ebeling W., Hertz H.G. Theorie der Electrolyte. Springer Verlag, Leipzig, 1971, p. 558.

[8] Yukhnovsky I.R., Holovko M.F. The statistical theory of the classical equilibrium systems. Naukova Dumka, Kiev, 1980, p. 376, (in Russian).

[9] Golovko M.F., Yukhnovsky I.R. Approaches to the many-body theory of dense ion-dipole plasma: Application to ionic solvation. In: The chemical physics of solvation. Elsevier, Amsterdam, vol. A, p. 207-262, 1985.

[10] Holovko M.F., Sovyak E.M. On taking account of interaction in the statistical theory of electrolyte solutions. // Cond. Matt. Phys., 1995, vol. 6, p. 49-78.

[11] Wertheim M.S. Fluids with highly directional attractive forces. III, IV. // J. Stat. Phys., 1986, vol. 42, No 3/4, p. 459-476, 477-492. 
[12] Wertheim M.S. Perturbation theory of polymerization. // J. Chem. Phys., 1987, vol. 87, p. 7323-7331.

[13] Kalyuzhnyi Yu.V., Stell G., Holovko M.F. Analytical solution of the multidensity OZ equation for polymerizing fluid. // Chem. Phys. Let., 1995, vol. 235, No 6, p. 335-369.

[14] Chang J., Sandler S.J. The correlation functions of hard-sphere chain fluids. // J. Chem. Phys, 1995, vol. 102, No 1, p. 437-449.

[15] Nefedov V.D., Texter E.N., Toropova M.A. Radiochemistry, Vischaja Schkola, Moscow, 1987 (in Russian).

[16] Holovko M.F., Kalyuzhnyi Yu.V. Studies of aqueous solution of polynuclear ions by the multidensity integral equation theory in polymer hypernetted chain approximation. (in preparation).

\title{
НОВІ АСПЕКТИ В ТЕОРІї ВОДНИХ РОЗЧИНІВ ЕЛЕКТРОЛІТІВ: ЕФЕКТИ КАТІОННОГО ГІДРОЛІЗУ ТА УТВОРЕННЯ БАГАТОЯДЕРНИХ ІОНІВ
}

\begin{abstract}
М.Ф.Головко
У творення багатоядерних іонів у водних розчинах електролітів імітується асоціативною міжкатіонною взаємодією. На основі багатогустинного асоціативного формалізму побудована статистична теорія цієі іонної моделі. Теорія передбачає значний вплив утворення багатоядерних іонів на термодинамічні та структурні властивості розчинів електролітів в широкій концентраційній області, включаючи область сильно розведених розчинів.
\end{abstract}

